\section{OPEN ACCESS}

Edited by:

Leonardo Alexandre Peyré-Tartaruga, Federal University of Rio Grande do Sul (UFRGS), Brazil

Reviewed by:

Rochelle Rocha Costa Federal University of Rio Grande do

Sul (UFRGS), Brazil

Patrick Christian Even,

Physiologie de la Nutrition et du Comportement Alimentaire (PNCA),

France

${ }^{*}$ Correspondence: Sidney B. Peres sbperes@uem.br

Specialty section

This article was submitted to Integrative Physiology, a section of the journal Frontiers in Physiology

Received: 30 October 2017

Accepted: 12 January 2018

Published: 06 February 2018

Citation:

Picoli CC, Romero PVS, Gilio GR, Guariglia DA, Tófolo LP, de Moraes $S M F$, Machado FA and Peres SB (2018) Peak Velocity as an Alternative

Method for Training Prescription in Mice. Front. Physiol. 9:42. doi: 10.3389/fphys.2018.00042

\title{
Peak Velocity as an Alternative Method for Training Prescription in Mice
}

\author{
Caroline de Carvalho Picoli ${ }^{1}$, Paulo Vitor da Silva Romero ${ }^{1}$, Gustavo R. Gilio', \\ Débora A. Guariglia ${ }^{1}$, Laize P. Tófolo ${ }^{1}$, Solange M. F. de Moraes ${ }^{2}$, Fabiana A. Machado ${ }^{1}$ \\ and Sidney B. Peres ${ }^{2 *}$
}

${ }^{1}$ Department of Physical Education, State University of Maringá, Paraná, Brazil, ${ }^{2}$ Department of Physiological Sciences, State University of Maringá, Paraná, Brazil

Purpose: To compare the efficiency of an aerobic physical training program prescribed according to either velocity associated with maximum oxygen uptake $\left(\mathrm{VVO}_{2 \max }\right)$ or peak running speed obtained during an incremental treadmill test $\left(V_{\text {peak_k }}\right)$ in mice.

Methods: Twenty male Swiss mice, 60 days old, were randomly divided into two groups with 10 animals each: 1. group trained by $\mathrm{VVO}_{2 \max }\left(\mathrm{GVO}_{2}\right)$, 2. group trained by $\mathrm{V}_{\text {peak_K}}$ (GVP). After the adaptation training period, an incremental test was performed at the beginning of each week to adjust training load and to determine the amount of $\mathrm{VO}_{2}$ and $\mathrm{VCO}_{2}$ fluxes consumed, energy expenditure (EE) and run distance during the incremental test. Mice were submitted to 4 weeks of aerobic exercise training of moderate intensity (velocity referring to $70 \%$ of $\mathrm{VVO}_{2 \max }$ and $\mathrm{V}_{\text {peak }} \mathrm{K}$ ) in a programmable treadmill. The sessions lasted from 30 to $40 \mathrm{~min}$ in the first week, to reach $60 \mathrm{~min}$ in the fourth week, in order to provide the mice with a moderate intensity exercise, totaling 20 training sessions.

Results: Mice demonstrated increases in $V_{2} \max \left(\mathrm{ml} \cdot \mathrm{kg}^{-1} \cdot \mathrm{min}^{-1}\right)\left(\mathrm{GVO}_{2}=49.1 \%\right.$ and GVP $=56.2 \%), V_{\text {peak_k }}\left(\mathrm{cm} \cdot \mathrm{s}^{-1}\right)\left(\mathrm{GVO}_{2}=50.9 \%\right.$ and $\left.\mathrm{GVP}=22.3 \%\right)$, EE $\left(\mathrm{ml} \cdot \mathrm{kg}^{-0,75} \cdot \mathrm{min}^{-1}\right)\left(\mathrm{GVO}_{2}=39.9 \%\right.$ and $\left.\mathrm{GVP}=51.5 \%\right)$, and run distance $(\mathrm{cm})$ $\left(\mathrm{GVO}_{2}=43.5 \%\right.$ and $\left.\mathrm{GVP}=33.4 \%\right)$, after 4 weeks of aerobic training (time effect, $P$ $<0.05)$; there were no differences between the groups.

Conclusions: $\mathrm{V}_{\text {peak_K}} \mathrm{K}$, as well as $\mathrm{VVO}_{2 \max }$, can be adopted as an alternative test to determine the performance and correct prescription of systemized aerobic protocol training to mice.

Keywords: treadmill, incremental test, running, training programs, exercise test

\section{INTRODUCTION}

The prescription of physical training in mice presents a great challenge owing to the large variety of available testing protocols (Kemi et al., 2002; Ferreira et al., 2007; Ayachi et al., 2016). In particular, running protocols are often used in research involving small rodents because they generally allow the intensity and volume of physical training to be manipulated more easily (Kregel et al., 2007).

Most of the available protocols are adapted from those applied and already tested for prescribed training in humans (Ayachi et al., 2016). Therefore, some variables used to predict human 
performance, such as maximal oxygen uptake $\left(\mathrm{VO}_{2 \max }\right)$, velocity associated with its occurrence $\left(\mathrm{vVO}_{2 \max }\right)$, thresholds related to blood lactate response (lactate threshold, anaerobic threshold, and maximal lactate steady state [MLSS]), and maximum running speed $\left(\mathrm{V}_{\text {peak }}\right)$ (Morgan et al., 1989; Bassett and Howley, 2000; Stratton et al., 2009) have also been used in several animals studies with the aim of reproducing the models already developed to evaluate and monitor training performance and prescription in humans (Carvalho et al., 2005; Ferreira et al., 2007; Manoel et al., 2017).

The most classical training prescription method for aerobic training in humans is based on tests using $\mathrm{VO}_{2 \max }$ (ACSM, 2011), which represents the highest rate at which oxygen is extracted in the lungs, transported, and used by the body during maximal exercise (Bassett and Howley, 2000), however, it cannot considered the best predictor of performance (Noakes et al., 1990). In order to more accurately predict endurance performance, $\mathrm{vVO}_{2 \max }$ has emerged and is defined as the minimum velocity at which $\mathrm{VO}_{2 \max }$ is reached in an incremental exercise protocol (Daniels et al., 1984; Morgan et al., 1989; Billat et al., 1994, 1999; Buchheit et al., 2010; Rodrigues et al., 2017).

Although $\mathrm{VO}_{2 \max }$ and $\mathrm{vVO}_{2 \max }$ are consolidated variables to predict performance and to monitor and prescribe aerobic training, their determination requires the use of gas analyzers and a team of researchers familiarized with such instruments, periodic calibration, and high cost. This is true whether the tests are performed in humans or rodents (Fernando et al., 1993; Wisløff et al., 2001). Therefore, reliable and less costly alternatives have been tested and validated for training prescription, such as peak running speed $\left(\mathrm{V}_{\text {peak }}\right)$, defined as the maximum velocity reached during an incremental test (Machado et al., 2013). This parameter is strongly correlated with endurance performance in human runners (di Prampero, 1986; Wisløff et al., 2001; Machado et al., 2007; Marcaletti et al., 2011) and presents a good correlation with $\mathrm{VO}_{2 \max }$.

However, a comparison of training prescriptions and monitoring according to $\mathrm{vVO}_{2 \max }$ and $\mathrm{V}_{\text {peak }}$ has not been performed and validated in mice. Such a study may help in reducing the cost and increasing the practicality of training prescription in this animal model. With the above in mind, the aim of this study was to compare the efficiency of an aerobic physical training program prescribed according to either $\mathrm{vVO}_{2 \text { max }}$ or $\mathrm{V}_{\text {peak_K }}$ in mice. Our hypothesis was that $\mathrm{V}_{\text {peak_K}}$, as well as $\mathrm{vVO}_{2 \max }$, can be used for training prescription to mice, with the advantage of not requiring expensive equipment.

\section{MATERIALS AND METHODS}

\section{Animals and Experimental Design}

Twenty Swiss male mice, 60 days old, acquired from the State University of Maringá (UEM), were maintained in individual polypropylene cages, lined with shavings and cleaned weekly, in an automated room for photoperiod control light-dark cycle $12 / 12$-h at $20 \pm 24^{\circ} \mathrm{C}$. The mice were allowed to feed (Nuvilab $\mathrm{Cr}{ }^{\circledR}$ ) and drink water ad libitum and food intake and body weight were measured weekly and at the end of the experiment. All procedures were previously approved by the
Ethics Committee in Animal Research of the State University of Maringá (UEM) (Maringá, Paraná, Brazil) (protocol $\mathrm{n}^{\circ}$ 033/2014).

Mice were randomly divided into two groups with 10 animals each: a group trained by $\mathrm{vVO}_{2 \max }\left(\mathrm{GVO}_{2}\right)$ and another group trained by $\mathrm{V}_{\text {peak_K }}(\mathrm{GVP})$. Mice were submitted to familiarization and adaptation to running exercise on a treadmill for 3 days, at an initial velocity of $8 \mathrm{~cm} \cdot \mathrm{s}^{-1}$ until reaching $16 \mathrm{~cm} \cdot \mathrm{s}^{-1}$, with an initial duration of $20 \mathrm{~min}$ until reaching $30 \mathrm{~min}$.

\section{Incremental Test}

The same incremental test was used for the determination of $\mathrm{VO}_{2 \max }, \mathrm{vVO}_{2 \max }$, and $\mathrm{V}_{\text {peak_K}}$, assessed on a motorized treadmill (Panlab ${ }^{\circledR}$, Barcelona, Spain), adapted from the protocol proposed by Machado et al. (2013) in humans. The warm-up period lasted 5-min at $10 \mathrm{~cm} \cdot \mathrm{s}^{-1}$, with an initial velocity of 19 $\mathrm{cm} \cdot \mathrm{s}^{-1}$, followed by an increase of $9 \mathrm{~cm} \cdot \mathrm{s}^{-1}$, every $3 \mathrm{~min}$ until exhaustion, which was characterized by the animal incapacity to keep running in the final third of the streak for more than $10 \mathrm{~s}$ (Kregel et al., 2007), $0^{\circ}$ slope. The speed unit used was $\mathrm{cm} \cdot \mathrm{s}^{-1}$ (Kurauti et al., 2016) and the training load was adjusted at the beginning of each training week and the group mean used to determine training intensity.

\section{Determination of $\mathrm{VO}_{2 \max }$ and $\mathrm{VVO}_{2 \max }$}

During the incremental test, gas chamber was collected to determine the $\mathrm{VO}_{2 \max }$ by an air flow control system that allows fine regulation by the LE450 Panlab ${ }^{\circledR}$ gas analyzer (Barcelona, Spain) to determine the amount of $\mathrm{VO}_{2}$ and $\mathrm{VCO}_{2}$ fluxes consumed, energy expenditure (EE) and run distance during the incremental test; the equipment was calibrated weekly. Before initiating the maximal incremental test, mice remained at rest for 5 min to determine the resting $\mathrm{VO}_{2}\left(\mathrm{VO}_{2 \text { rep }}\right)$ (Billat et al., 2005; Machado et al., 2013). $\mathrm{VO}_{2 \max }$ was expressed in values related to body mass from an allometric adjustment equal to 1 (Taylor et al., 1955, 1981).

Exercise intensity data and $\mathrm{VO}_{2 \max }$ were recorded every second (METABOLISM software, Pan Lab/Harvard Instruments, Spain) and monitored by an external researcher who visually determined the highest $\mathrm{VO}_{2}$ reached during the test considered the $\mathrm{VO}_{2 \max }$ (McLaughlin et al., 2010), measured at an average of 5-s intervals. Therefore, $\mathrm{vVO}_{2 \max }$ was determined as the minimal velocity at which the highest $\mathrm{VO}_{2 \max }$ occurred (Billat et al., 1994, 1999).

\section{Determination of $\mathbf{V}_{\text {peak_k }}$}

$\mathrm{V}_{\text {peak }}$ was considered the peak running speed obtained during the incremental treadmill test (Machado et al., 2013); if the last stage during the test was not completed, the $V_{\text {peak_K }}$ was calculated on the part-time achieved using the equation proposed by Kuipers et al. (2003): $\mathrm{V}_{\text {peak_K }}=(\mathrm{V}+\mathrm{t} / \mathrm{T}$ x speed increase), where $\mathrm{V}$ is the corresponding velocity of the last completed stage $\left(\mathrm{cm} \cdot \mathrm{s}^{-1}\right)$, $\mathrm{t}$ the time (s) of the uncompleted step and, $\mathrm{T}$ the completed step (180s). 


\section{Training Protocol}

Mice were submitted to 4 weeks of aerobic exercise training in a programmable treadmill (Inbrasport, Porto Alegre, Brazil) adapted with a support to accommodate 10 mice simultaneously. The training intensity was established at $70 \%$ of the maximum speed reached during the incremental test for the determination of the variables, $\mathrm{vVO}_{2 \max }$ and $\mathrm{V}_{\text {peak_K}}$, for the $\mathrm{GVO}_{2}$ and GVP groups, respectively. The $70 \%$ maximum speed of training correspond to a moderate exercise session since it presents a high correlation with $\mathrm{VO}_{2 \max }$. (Manoel et al., 2017).Training sessions began at $6 \mathrm{a} . \mathrm{m}$. (lights on), consisting of 1 session per day, 5 times a week (Monday to Friday). The sessions lasted from 30 to $40 \mathrm{~min}$ in the first week, from 35 to $50 \mathrm{~min}$ in the second week, and 50 to $60 \mathrm{~min}$ in the third week, reaching $60 \mathrm{~min}$ in the fourth week, in order to submit mice to a moderate intensity exercise, totaling 20 training sessions; the weekly test was considered a training session.

\section{Data Analysis}

The sample size was calculated using the G-power software (v.3.1.9.2) (Faul et al., 2007), which demonstrated the necessity of a sample of 11 mice per group for the main variable $V_{\text {peak_K}}$; the average effect size was set at $0.25, P<0.05$ and $80 \%$ power for ANOVA analysis with effect and interaction. All statistical analyses were performed using the Statistica software (v.10, StatSoft Inc., Tulsa, OK, USA). Data are presented as mean \pm standard deviation $(S D)$. Shapiro-Wilk test was performed to verify data normality. Comparison of the pre-and post- training was made by mixed ANOVA for repeated measurements. To locate the differences, Tukey post-hoc was applied if significance was observed. The significance level was set at $P<0.05$. The effect size was calculated using Cohen equation $(d=$ $\mathrm{M}_{1}-\mathrm{M}_{2} / S D$ pooled $)$ comparing post- vs. pre-training period of $\mathrm{vVO}_{2 \max }$ and $\mathrm{V}_{\text {peak_K}}$. Values of $0.2,0.5$, and 0.8 indicated a small, medium and large average effect, respectively (Cohen, 1988).

\section{RESULTS}

Table 1 shows significant differences in body weight (g), food intake (g) and food intake corrected by body weight after 4 weeks of training (time effect, $P<0.05$ ). Mice showed increased body weight $(\mathrm{g})$ but a reduction of food intake $(\mathrm{g})$ over time in both groups. The food intake after 4 weeks showed a marked reduction of GVP group in comparison to GVO2, although not significant.

Table 2 demonstrates that 4 weeks of training promoted increases in $\mathrm{VO}_{2 \max }\left(\mathrm{ml} \cdot \mathrm{kg}^{-1} \cdot \mathrm{min}^{-1}\right), \mathrm{vVO}_{2 \max }\left(\mathrm{cm} \cdot \mathrm{s}^{-1}\right)$, $\mathrm{V}_{\text {peak_K }}\left(\mathrm{cm} \cdot \mathrm{s}^{-1}\right)$, EE $\left(\mathrm{ml} \cdot \mathrm{kg}^{-1} \cdot \mathrm{min}^{-1}\right)$, run distance $(\mathrm{cm})$, and delta (\%) after 4 weeks of aerobic training (time effect, $P<0.05)$. Also, $\mathrm{GVO}_{2}$ group presented an interaction effect $(P<0.05)$, indicating the combination of group and time together influenced $\mathrm{vVO}_{2 \max }$. There were no differences between the groups in pre-or post-conditions (group effect).
In order to expand the data analysis beyond the descriptive statistic, we calculated the effect size comparing post- vs. pretraining period for $\mathrm{vVO}_{2 \max }$ and $\mathrm{V}_{\text {peak_K}}$ to assess the magnitude of findings; Figure 1 shows a large effect in both groups.

\section{DISCUSSION}

The aim of the present study was to compare the efficiency of an aerobic physical training program prescribed according to either $\mathrm{vVO}_{2 \max }$ and $\mathrm{V}_{\text {peak_K }}$ in mice. Our study demonstrates that both groups, $\mathrm{GVO}_{2}$ or GVP, improved $\mathrm{VO}_{2 \text { máx }}, \mathrm{vVO}_{2 \text { max., }}$, and $V_{\text {peak_K}}$ after 4 weeks of aerobic training. In addition, $V_{\text {peak_K }}$ has been proved a reliable method to prescribe and monitor exercise training. Moreover, training improved performance in both groups and increased $\mathrm{EE}$ and the run distance. To our knowledge, this is the first study to address the importance of $\mathrm{V}_{\text {peak_K }}$ in animal studies.

In the field of exercise physiology, there is great interest in determining the precise intensity of exercise as various research projects use animal models to study the effects of acute and chronic exercise. In this context, several methods to determine training load have been proposed (Kemi et al., 2002; Lerman et al., 2002; Ferreira et al., 2007).

Non-invasive tests report in the literature that use of $\mathrm{VO}_{2 \max }$ as the variable to predict the performance of mice diverge from incremental tests and training protocols. In this sense, establishing a consensus about the results is not possible due to a wide range of reported values (47-94 $\mathrm{ml} \cdot \mathrm{kg}^{-1} \cdot \mathrm{min}^{-1}$ ), according to the slope, magnitude of increase, volume, exhaustion criteria, lineages, age, physical condition, and sex of animals (Dohm et al., 1994; Scott and Houmard, 1994; Lightfoot et al., 2001; Wisløff et al., 2001; Kemi et al., 2002; Ayachi et al., 2016; Kurauti et al., 2016). A few studies have also focused on demonstrating that lineage and sex variations in mice may influence responses in aerobic power (Lightfoot et al., 2010; McLaughlin et al., 2010).

Considering that, it was conceivable that the use of $\mathrm{V}_{\text {peak_K }}$ would help to minimize the observed differences in $\mathrm{VO}_{2 \max }$ once the variable is more sensitive to changes in physical training in humans (Machado et al., 2013; Manoel et al., 2017). In our study, animals trained according to $\mathrm{vVO}_{2 \max }$ or $\mathrm{V}_{\text {peak_K }}$ had similar improvements in $\mathrm{VO}_{2 \max }$ and performance over time. In fact, $\mathrm{GVO}_{2}$ group presented a higher, although not significant, $\mathrm{V}_{\text {peak_K}}$. Despite the differences between the two prescription methods it is important to emphasize that the current study demonstrated that animals were able to improve aerobic capacity over time. From a metabolic point of view, other metabolic and structural adaptations may have occurred, hence allowing $\mathrm{V}_{\text {peak_K}}$-based protocol to be a trustworthy training model for rodents. Therefore, we hypothesize that $V_{\text {peak_K }}$ can also contribute to the prescription of exercise in different lineages, sex, and age groups of mice, since it has proved as a training-sensitive variable.

The determination of $\mathrm{V}_{\text {peak_K}}$, considering the equation proposed by Kuipers et al. (2003), allows evaluating aerobic power and monitoring the effects of training more reliable, as the 
TABLE 1 | Body weight, food intake and food intake/body weight in pre- and post-training.

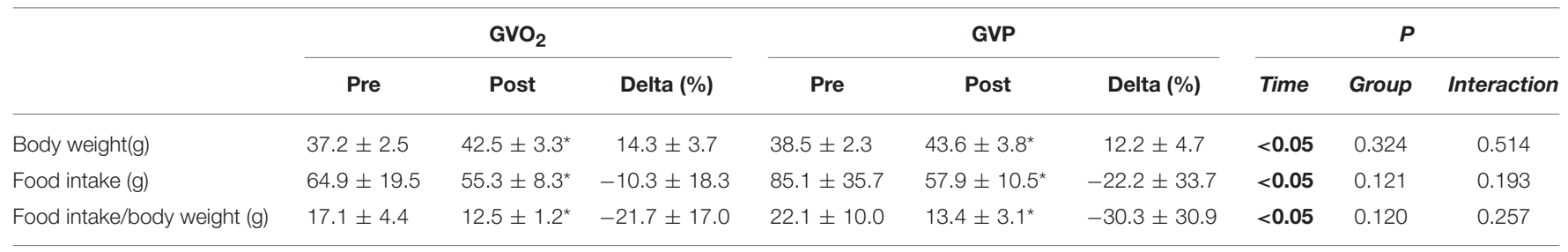

Values are presented as mean $\pm S D$. Delta percentage (\%) and P-values for time, group and interaction. ${ }^{*} P<0.05$ significantly different for time comparison (pre- vs. post-training period).

TABLE 2 | Pre- and post-training $\mathrm{VO}_{2 \max }, \mathrm{V}_{\text {peak_K}}, \mathrm{VVO}_{2 \max }$, EE and run distance.

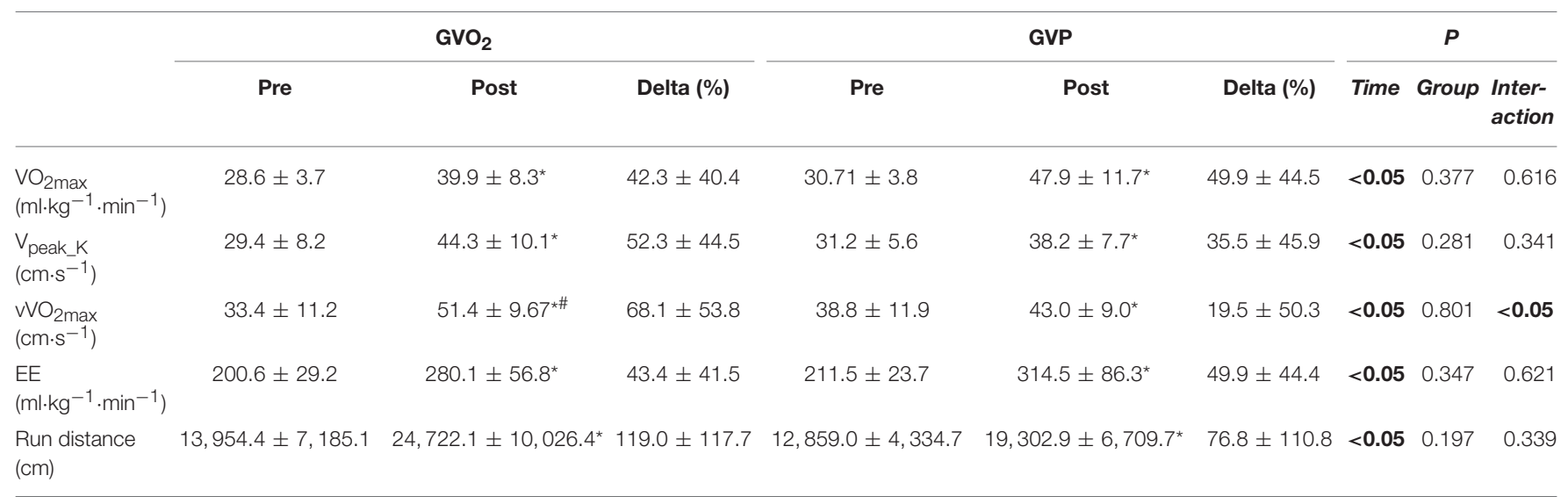

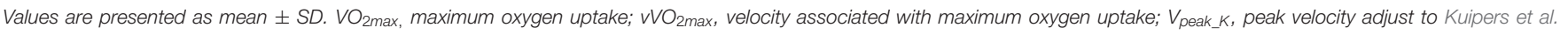
(2003); EE, energy expenditure. Delta percentage (\%) and P-values for time, group, and interaction analysis.

${ }^{\star} P<0.05$ significantly different for time comparison (pre- vs. post-training period).

${ }^{\#} P<0.05$ significantly different for interaction comparison (pre- vs. post-training period).

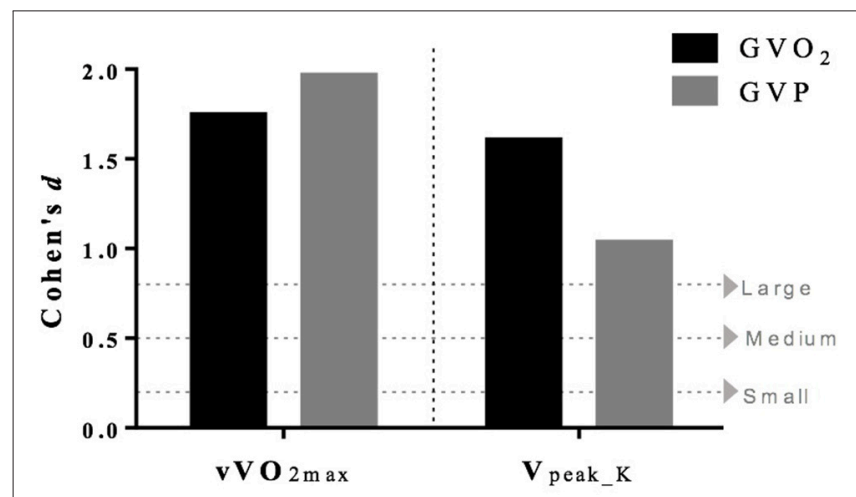

FIGURE 1 | Cohen's effect size comparing post- vs. pre-training period for vVO2max and Vpeak_k.

equation takes into account the last stage completed added to the product of the rate of increase and the fraction of the incomplete last stage, abolishing the influence of subjective judgments or overestimations (Machado et al., 2013). Furthermore, $V_{\text {peak_K }}$ is a low-cost, practical, and reliable parameter, which makes it a fundamental tool for future research in the field of exercise physiology, especially considering the known difficulties of assessing $\mathrm{VO}_{2 \max }$ in small rodents (Fernando et al., 1993; Scott and Houmard, 1994; Ayachi et al., 2016).

Although our research was carefully conducted, limitations and shortcomings are unavoidable. The content of muscle glycogen or correlation with blood levels of lactate were not verified during the training period. Also, the training was performed during the day (lights on), opposable to mice habits, which present a predominantly nocturnal behavior.

$\mathrm{V}_{\text {peak_K}} \mathrm{K}$ has practical implications for researchers aiming to collect data concerning the effects of training due to its low cost, which is made possible by the fact that it does not require any expensive equipment (i.e., gas analyzer). In summary, $\mathrm{V}_{\text {peak_K}} \mathrm{K}$ as well as $\mathrm{vVO}_{2 \max }$, can be adopted as an alternative test to determine the performance and correct prescription of a systemized aerobic protocol training to mice.

\section{AUTHOR CONTRIBUTIONS}

CP: Experiment design, execution of the experiments, writing; PR: Experiment design; GG: Execution of the experiments; DG: Performed statistical analysis; LT: VO2 analysis: SdM: Discussion of data; FM: Conceive the experiments, discussion of data, writing; SP: Concept of the paper, writing. 


\section{REFERENCES}

ACSM's (2011). "ACSM's cardiorespiratory fitness: field and submaximal exercise test," in ACSM's Health-Related Physical Fitness Assessment Manual, 4th Edn, ed L. A. Kamisky (Baltimore, MD: Wolters Kluwer), 135-150.

Ayachi, M., Niel, R., Momken, I., Billat, V. L., and Mille-Hamard, L. (2016). Validation of a ramp running protocol for determination of the true VO2max in Mice. Front. Physiol. 7:372. doi: 10.3389/fphys.2016.00372

Bassett, D. R., and Howley, E. T. (2000). Limiting factors for maximum oxygen uptake and determinants of endurance performance. Med. Sci. Sports Exerc. 32, 70-84. doi: 10.1097/00005768-200001000-00012

Billat, V. L., Flechet, B., Petit, B., Muriaux, G., and Koralsztein, J. P. (1999). Interval training at $\mathrm{VO} 2 \mathrm{max}$ : effects on aerobic performance and overtraining markers. Med. Sci. Sports Exerc. 31, 156-163. doi: 10.1097/00005768-199901000-00024

Billat, V. L., Mouisel, E., Roblot, N., and Melki, J. (2005). Inter- and intrastrain variation in mouse critical running speed. J. Appl. Physiol. 98, 1258-1263. doi: 10.1152/japplphysiol.00991.2004

Billat, V., Renoux, J. C., Pinoteau, J., Petit, B., and Koralsztein, J. P. (1994). Reproducibility of running time to exhaustion at VO2max in subelite runners. Med. Sci. Sports Exerc. 26, 254-257. doi: 10.1249/00005768-199402000-00018

Buchheit, M., Chivot, A., Parouty, J., Mercier, D., Al Haddad, H., Laursen, P. B., et al. (2010). Monitoring endurance running performance using cardiac parasympathetic function. Eur. J. Appl. Physiol. 108, 1153-1167. doi: 10.1007/s00421-009-1317-x

Carvalho, J. F., Masuda, M. O., and Pompeu, F. A. M. S. (2005). Method for diagnosis and control of aerobic training in rats based on lactate threshold. Comp. Biochem. Physiol. A Mol. Integr. Physiol. 140, 409-413. doi: 10.1016/j.cbpb.2004.12.002

Cohen, J. (1988). Statistical Power Analysis for the Behavioral Sciences 2nd Edn. Hillsdale, NJ: Lawrence Earlbaum Associates.

Daniels, J., Scardina, N., Hayes, J., and Foley, P. (1984). "Elite and subelite female middle- and long-distance runners," in Sport and Elite Performers, ed D. M. Landers (Champaign, IL: Human Kinetics), 57-72.

di Prampero, P. E. (1986). The anaerobic threshold concept: a critical evaluation. Adv. Cardiol. 35, 24-34. doi: 10.1159/000413436

Dohm, M. R., Richardson, C. S., and Garland, T. (1994). Exercise physiology of wild and random-bred laboratory house mice and their reciprocal hybrids. Am. J. Physiol. 267, R1098-R1108. doi: 10.1152/ajpregu.1994.267.4.R1098

Faul, F., Erdfelder, E., Lang, A., and Buchner, A. (2007). G*Power 3: a flexible statistical power analysis program for the social, behavioral, and biomedical sciences. Behav. Res. Methods 39, 175-191. doi: 10.3758/BF03193146

Fernando, P., Bonena, and Hoffman-Goetz, L. (1993). Predicting submaximal oxygen consumption during treadmill running in mice. Can. J. Physiol. Pharmacol. 71, 854-857. doi: 10.1139/y93-128

Ferreira, J. C. B., Rolim, N. P. L., Bartholomeu, J. B., Gobatto, C. A., Kokubun, E., and Brum, P. C. (2007). Maximal lactate steady state in running mice: effect of exercise training. Clin. Exp. Pharmacol. Physiol. 34, 760-765. doi: 10.1111/j.1440-1681.2007.04635.x

Kemi, O. J., Loennechen, J. P., Wisløff, U., Ellingsen, Ø., Johan, O., Loennechen, J. P., et al. (2002). Intensity-controlled treadmill running in mice : cardiac and skeletal muscle hypertrophy. J. Appl. Physiol. 93, 1301-1309. doi: 10.1152/japplphysiol.00231.2002

Kregel, K. C., Allen, D. L., Booth, F. W., Fleshner, M., Henriksen, E. J., Musch, T. I., et al. (2007). Resource Book for the Design of 302 Animal Exercise Protocols, $1 s t$ Edn. Bethesda, MD: American Physiological Society.

Kuipers, H., Rietjens, G., Verstappen, F., Schoenmakers, H., and Hofman, G. (2003). Effects of stage duration in incremental running tests on physiological variables. Int. J. Sports Med. 24, 486-491. doi: 10.1055/s-2003-42020

Kurauti, M. A., Freitas-Dias, R., Ferreira, S. M., Vettorazzi, J. F., Nardelli, T. R., Araujo, H. N., et al. (2016). Acute exercise improves insulin clearance and increases the expression of insulin-degrading enzyme in the liver and skeletal muscle of swiss mice. PLOS ONE 11:e0160239. doi: 10.1371/journal.pone.0160239

Lerman, I., Harrison, B. C., Freeman, K., Hewett, T. E., Allen, D. L., Robbins, J., et al. (2002). Genetic variability in forced and voluntary endurance exercise performance in seven inbred mouse strains. J. Appl. Physiol. 92, 2245-2255. doi: 10.1152/japplphysiol.01045.2001
Lightfoot, J. T., Leamy, L., Pomp, D., Turner, M. J., Fodor, A. A., Knab, A., et al. (2010). Strain screen and haplotype association mapping of wheel running in inbred mouse strains. J. Appl. Physiol. 109, 623-634. doi: 10.1152/japplphysiol.00525.2010

Lightfoot, J. T., Turner, M. J., Debate, K. A., and Kleeberger, S. R. (2001). Interstrain variation in murine aerobic capacity. Med. Sci. Sports Exerc. 33, 2053-2057. doi: 10.1097/00005768-200112000-00012

Machado, F. A., Guglielmo, L. G., and Denadai, B. S. (2007). Effect of the chronological age and sexual maturation on the time to exhaustion at maximal aerobic speed. Web Sci. 36, 21-30. doi: 10.1590/2179-325520143 630018

Machado, F. A., Kravchychyn, A. C. P., Peserico, C. S., da Silva, D. F., and Mezzaroba, P. V. (2013). Incremental test design, peak "aerobic" running speed and endurance performance in runners. J. Sci. Med. Sport 16, 577-582. doi: 10.1016/j.jsams.2012.12.009

Manoel, F. A., da Silva, D. F., Roberto Perrout de Lima, J., and Andrade Machado, F. (2017). Peak velocity and its time limit are as good as the velocity associated with VO2max for training prescription in runners. Sport. Med. Int. Open 1, 8-15. doi: 10.1055/s-0042-119951

Marcaletti, S., Thomas, C., and Feige, J. N. (2011). Exercise performance tests in mice. Curr. Protoc. Mouse Biol. 1, 141-154. doi: 10.1002/9780470942390.mo100160

McLaughlin, J. E., Howley, E. T., Bassett, D. R., Thompson, D. L., and Fitzhugh, E. C. (2010). Test of the classic model for predicting endurance running performance. Med. Sci. Sports Exerc. 42, 991-997. doi: 10.1249/MSS.0b013e3181c0669d

Morgan, D. W., Baldini, F. D., Martin, P. E., and Kohrt, W. M. (1989). Ten kilometer performance and predicted velocity at VO2max among well-trained male runners. Med. Sci. Sports Exerc. 21, 78-83. doi: 10.1249/00005768-198902000-00014

Noakes, T. D., Myburgh, K. H., and Schall, R. (1990). Peak treadmill running velocity during the VO2 max test predicts running performance. J. Sports Sci. 8, 35-45. doi: 10.1080/02640419008732129

Rodrigues, N. A., Torsoni, A. S., Fante, T., Dos Reis, I. G., Gobatto, C. A., and Manchado-Gobatto, F. B. (2017). Lactate minimum underestimates the maximal lactate steady state in swimming mice. Appl. Physiol. Nutr. Metab. 42, 46-52. doi: 10.1139/apnm-2016-0198

Scott, B., and Houmard, J. (1994). Peak running velocity is highly related to distance running performance. Int. J. Sports Med. 15, 504-507. doi: 10.1055/s-2007-1021095

Stratton, E., O’Brien, B. J., Harvey, J., Blitvich, J., McNicol, A. J., Janissen, D., et al. (2009). Treadmill velocity best predicts 5000-m run performance. Int. J. Sports Med. 30, 40-45. doi: 10.1055/s-2008-1038761

Taylor, C. R., Maloiy, G. M., Weibel, E. R., Langman, V. A., Kamau, J. M., Seeherman, H. J., et al. (1981). Design of the mammalian respiratory system. Respir. Physiol. 44, 25-37. doi: 10.1016/0034-5687(81)90075-X

Taylor, H. L., Buskirk, E., and Henschel, A. (1955). Maximal oxygen intake as an objective measure of cardio-respiratory performance. J. Appl. Physiol. 8, 73-80. doi: 10.1152 /jappl.1955.8.1.73

Wisløff, U., Helgerud, J., Kemi, O. J., and Ellingsen, O. (2001). Intensity-controlled treadmill running in rats: $\mathrm{VO}(2 \mathrm{max})$ and cardiac hypertrophy. Am. J. Physiol. Heart Circ. Physiol. 280, H1301-H1310. doi: 10.1152/ajpheart.2001.280.3.H1301

Conflict of Interest Statement: The authors declare that the research was conducted in the absence of any commercial or financial relationships that could be construed as a potential conflict of interest.

The reviewer RRC and handling Editor declared their shared affiliation.

Copyright (c) 2018 Picoli, Romero, Gilio, Guariglia, Tófolo, de Moraes, Machado and Peres. This is an open-access article distributed under the terms of the Creative Commons Attribution License (CC BY). The use, distribution or reproduction in other forums is permitted, provided the original author(s) and the copyright owner are credited and that the original publication in this journal is cited, in accordance with accepted academic practice. No use, distribution or reproduction is permitted which does not comply with these terms. 